\title{
AMC-based planar antenna with low-profile and broad CP beamwidth
}

\author{
Jiadong Lin ${ }^{\text {a)}}$, Zuping Qian, and Wenquan Cao \\ College of Communications Engineering, PLA University of Science and Technology, \\ Nanjing 210007, China \\ a)a851977701@126.com
}

\begin{abstract}
A low-profile circularly-polarized (CP) antenna with broad axialratio (AR) beamwidth is presented by loading with artificial magnetic conductor (AMC). The antenna consists of a couple of modified dipoles with four crossed symmetrical fan-shaped arms and a magneto-electric (ME) dipole equipped to broaden AR beamwidth. One AMC consists of $5 \times 5$ units is integrated to reduce the backward scattering wave and simultaneously ensures a low profile. The measured results of one fabricated type reveal that the AMC-integrated antenna accomplishes a wide 3-dB AR beamwidth $\left(>135^{\circ}\right)$ and exhibits a low-profile property with the height of $0.054 \lambda_{0}$, which can be applied in the BeiDou Satellite System (BDS).
\end{abstract}

Keywords: artificial magnetic conductor (AMC), broad CP beamwidth, low-profile, microstrip antenna

Classification: Microwave and millimeter-wave devices, circuits, and modules

\section{References}

[1] X. L. Bao and M. J. Ammann: "Dual-frequency dual circularly-polarized patch antenna with wide beamwidth," IET Electron. Lett. 44 (2008) 1233 (DOI: 10. 1049/el:20082284).

[2] Nasimuddin, et al.: "A wide-beam circularly polarized asymmetric-microstrip antenna,” IEEE Trans. Antennas Propag. 63 (2015) 3764 (DOI: 10.1109/TAP. 2015.2438397).

[3] Y. Kazama, et al.: "Vertical polarization single-feed dual-frequency microstrip antenna with an arc-shaped slot," IEICE Electron. Express 2 (2005) 60 (DOI: 10.1587/elex.2.60).

[4] S. X. Ta and I. Park: "Crossed dipole loaded with magneto-electric dipole for wideband and wide-beam circularly polarized radiation," IEEE Antennas Wireless Propag. Lett. 14 (2015) 358 (DOI: 10.1109/LAWP.2014.2363944).

[5] L. Chen, et al.: "Wideband circularly polarized microstrip antenna with wide beamwidth," IEEE Antennas Wireless Propag. Lett. 13 (2014) 1577 (DOI: 10. 1109/LAWP.2014.2341241).

[6] K. M. Mak and K. M. Luk: "A circularly polarized antenna with wide axial ratio beamwidth," IEEE Trans. Antennas Propag. 57 (2009) 3309 (DOI: 10. 1109/TAP.2009.2029370). 
2009.2014527).

[8] X. Y. Liu, et al.: "A planar Windmill-like broadband antenna equipped with artificial magnetic conductor for off-body communications," IEEE Antennas Wireless Propag. Lett. 15 (2016) 64 (DOI: 10.1109/LAWP.2015.2429683).

[9] L. Wang, et al.: "Design of a new printed dipole antenna using in high latitudes for Inmarsat,” IEEE Antennas Wireless Propag. Lett. 10 (2011) 358 (DOI: 10. 1109/LAWP.2011.2146224).

[10] Y. Zhang, et al:: "Planar artificial magnetic conductors and patch antennas," IEEE Trans. Antennas Propag. 51 (2003) 2704 (DOI: 10.1109/TAP.2003. $817550)$.

\section{Introduction}

Antennas with broad circularly-polarized (CP) beamwidth radiation can be used in many applications such as RFID, base station communication [1], especially Global Navigation Satellite Systems (GNSS) like BeiDou Satellite System (BDS). For the fixture on the satellite, antennas are required to have the characteristics of low profile and light weight [2] and [3]. However, requirement of wide-beamwidth $\mathrm{CP}$ radiation make antennas complicated and difficult to be manufactured. Many studies have been done to broaden the $\mathrm{CP}$ beamwidth of antennas $[4,5,6]$. The work presented in [4] adopted the metallic cavity with a size of $0.72 \times 0.72 \times 0.18$ $\lambda_{0}{ }^{3}$ to realize a $3-\mathrm{dB}$ axial ratio (AR) beamwidth of more than $130^{\circ}$. A wideband $\mathrm{CP}$ microstrip antenna loaded with a folded ground plane for a 3-dB AR beamwidth of more than $120^{\circ}$ was designed in [5]. In [6], a magnetic dipole structure and a electric dipole were assembled to form a CP antenna with wide AR beamwidth. However, due to the lack of techniques to simplify the structure and reduce the profile, most of the aforementioned antennas have a huge volume and a high profile. Thus antennas with broad CP beamwidth and low profile are required.

With the development of artificial magnetic conductor (AMC) or high impedance surface (HIS), antennas with low-profile property have been studied using these periodic structures to replace conventional PEC ground planes. A dual-band wearable textile antenna on an electromagnetic band-gap (EBG) substrate is investigated in [7]. In [8], a planar windmill-like broadband antenna equipped with AMC is studied. As the AMC/HIS can guide the electromagnetic wave into the upper-half space and owns the characteristic of in-phase reflection, a radiating structure can be placed quite close to the AMC/HIS structure without affecting its impedance bandwidth and $\mathrm{CP}$ characteristics.

In this letter, a novel low-profile microstrip antenna with broad CP beamwidth is realized based on AMC. Generally antennas with broad CP beamwidth need a metallic cavity to guarantee unidirectional radiation, which makes antennas bulky and difficult to be fabricated. In this work, AMC array acting as an electromagnetic wave reflector is designed to replace the conventional metallic cavity of broad CP beamwidth antennas. Without destroying broad CP beamwidth characteristic, the AMC-based antenna has an amazing low profile, high gain and compact dimension. Simulator (HFSS) and then verified by measurements. The detailed design of 
proposed antenna is shown in Section II. The simulation and measurement results are studied in Section III. Finally, conclusions are elaborated in Section IV.

\section{Antenna configuration and analysis}

The structure of the proposed antenna is shown in Fig. 1, which consists of three layers, containing an AMC, a coaxial line, a magneto-electric (ME) dipole and a crossed dipole. The ME dipole is composed of four metallic plates, which are grounded via four metallic posts. In order to reduce the antenna profile, both the four metallic plates of the ME dipole and the crossed dipole were etched on a Rogers RO4003 substrate with a thickness of $H_{1}=0.508 \mathrm{~mm}$, a relative permittivity of 3.38 and a loss-tangent of 0.0027 . The crossed double-sided printed dipole with vacant quarter-ring is used to realize $\mathrm{CP}$ property. Once the magnetic and the electric dipoles are coupled from the cross dipole simultaneously with proper amplitudes and phases, the CP radiating pattern can be broaden in the broadside direction. The selected parameters for the optimized antenna are listed as follows: $W_{\mathrm{p}}=57 \mathrm{~mm}, R_{\mathrm{p}}=6 \mathrm{~mm}, D_{1}=60^{\circ}, D_{\mathrm{p}}=3 \mathrm{~mm}, S_{\mathrm{p}}=7 \mathrm{~mm}, L_{\mathrm{d}}=20 \mathrm{~mm}, \mathrm{D}=$ $25^{\circ}, \quad R_{1}=4 \mathrm{~mm}, \quad R_{2}=3.7 \mathrm{~mm}, W_{\mathrm{r}}=0.6 \mathrm{~mm}, W_{1}=14.1 \mathrm{~mm}, W_{2}=3 \mathrm{~mm}$, $W_{3}=1.9 \mathrm{~mm}$.

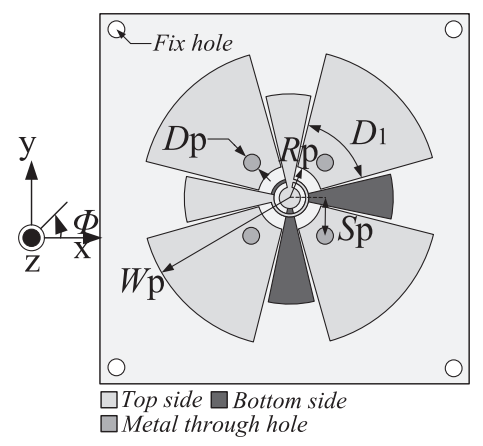

(a)

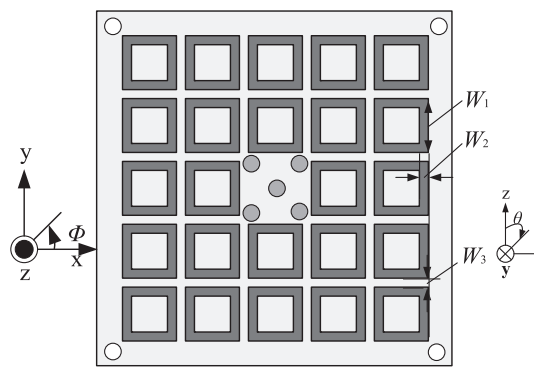

(c)

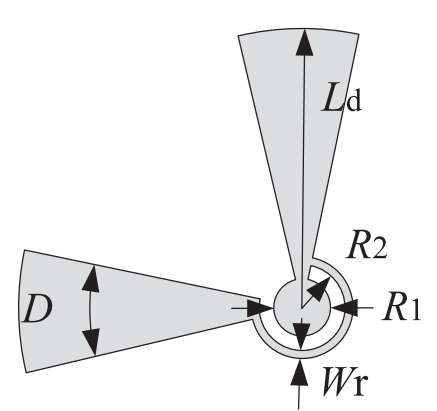

(b)

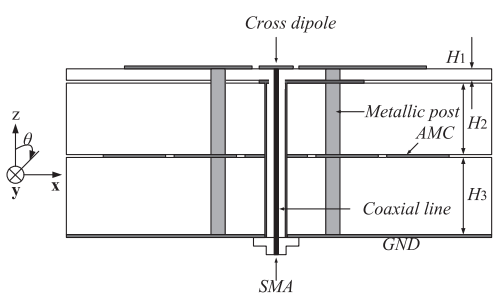

(d)

Fig. 1. Geometry of proposed antenna, (a) top view of radiator and geometry of the magneto-electric dipole, (b) geometry of the modified cross dipole, (c) geometry of the AMC, (d) crosssectional view of the AMC-based antenna.

As exhibited in Fig. 1(c) and Fig. 2(a), in order to further strengthen the forward radiation and reduce the profile, an AMC array acting as an electromagnetic wave reflector is designed to replace the conventional metallic cavity. The AMC array is made on one F4BM-2 substrate with thickness of $H_{3}=3 \mathrm{~mm}$, a relative permittivity of 3.55 . The $5 \times 5$ square-ring patches are printed on the top 


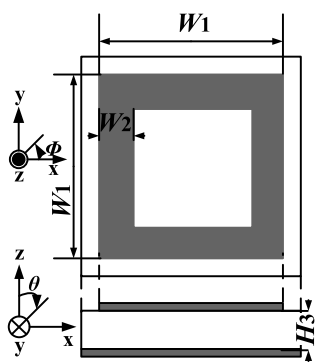

(a)
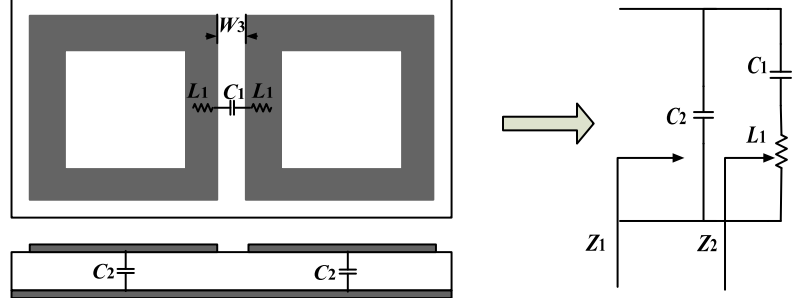

(b)

Fig. 2. (a) Configuration and (b) equivalent circuit of adjacent ringshaped AMC units.

surface of the substrate. The bottom surface is the metal ground. Though analyzing the working principle in [10], the EM field in AMC structure can be simplified to the circuit model given in Fig. 2(b). Thus, its total surface impedance can be expressed as

$$
Z_{0}(\omega)=Z_{1} / / Z_{2}=\frac{j\left(1-\omega^{2} C_{2} L_{1}\right)}{\omega\left(C_{1} C_{2} L_{1} \omega^{2}-C_{1}-C_{2}\right)}
$$

From (1), the resonant frequency of the construction can be calculated as

$$
f_{0}=\frac{1}{2 \pi} \sqrt{\frac{C_{1}+C_{2}}{C_{1} C_{2} L_{1}}}
$$

where $C_{1}, L_{1}, C_{2}$ are the capacitance between units, unit inductance, and capacitance between unit and ground plane, respectively.

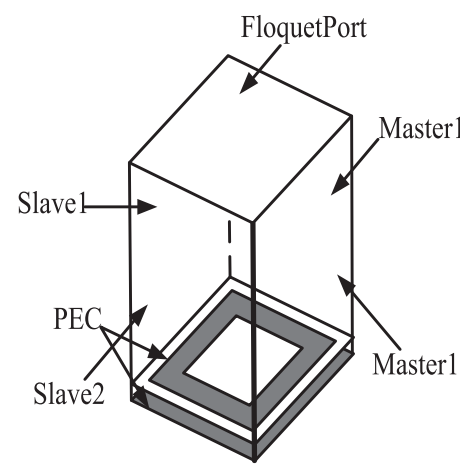

(a)

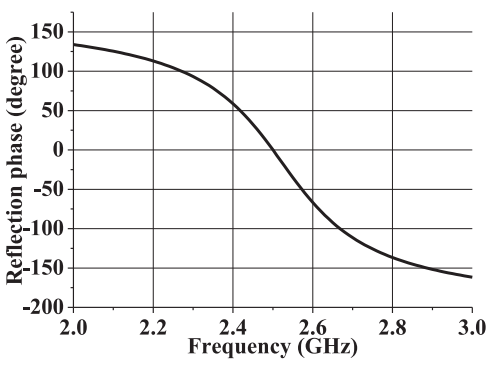

(b)

Fig. 3. (a) Model used for the simulation. (b) Reflection phase of the proposed AMC.

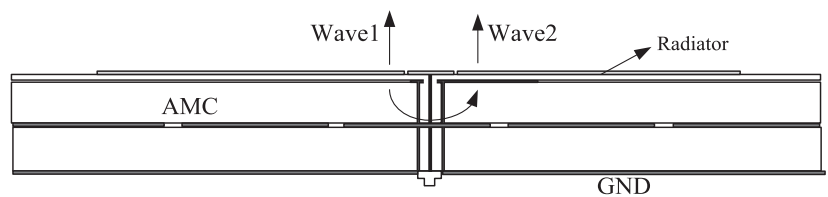

Fig. 4. Wave property of AMC based antenna.

As shown in Fig. 3(a), the model based on a floquet-port air-filled wave guide with master-slave boundary conditions is adopted for numerically computing the reflection phase of AMC. With reference to Fig. 3(b), the reflection phase of 
$2.5 \mathrm{GHz}$ is around $0^{\circ}$. Thus, the designed $\mathrm{AMC}$ can guarantee the transmitted wave (Wave1) and reflected wave (Wave2) in-phase at $2.5 \mathrm{GHz}$ as shown in Fig. 4. In order to obtain light weight, adequate toughness, and reasonable isolation, a piece of F4BM-2 substrate with a thickness of $H_{2}=3 \mathrm{~mm}$ is filled in the interlayer.

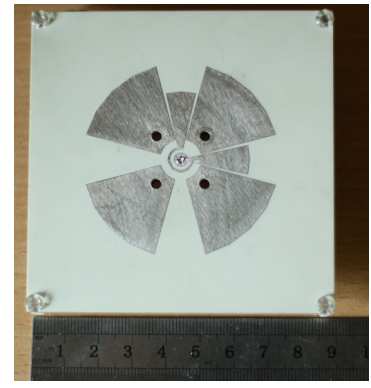

(a)

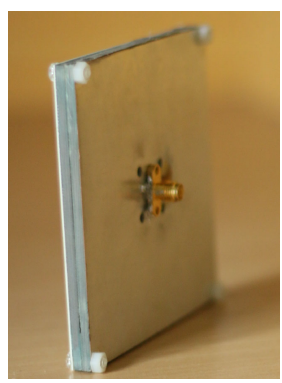

(b)

Fig. 5. Photograph of proposed antenna: (a) top view (b) back view.

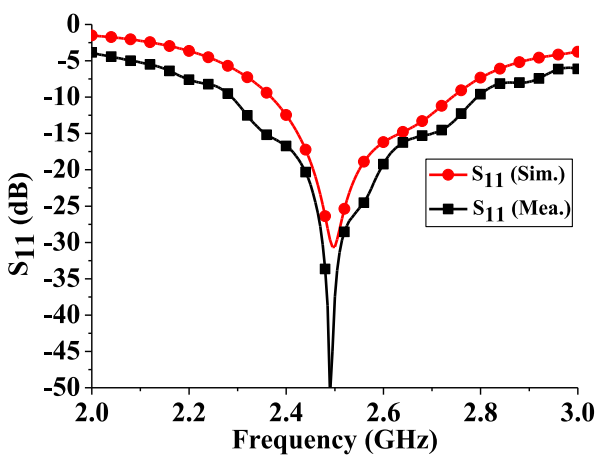

(a)

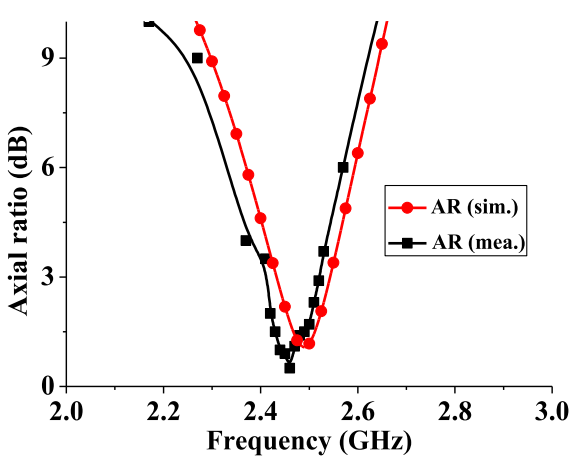

(b)

Fig. 6. Measurement and simulation: (a) $S_{11}$ and (b) AR values

\section{Experimental result and discussion}

The AMC-based antenna is fabricated to confirm the low profile and broad CP beamwidth property. Fig. 5 shows the photographs of the proposed antenna. The $5 \times 5$ AMC units were built on one side of an F4BM-2 substrate with a copper thickness $35 \mu \mathrm{m}$. The crossed dipole and four metallic plates of the ME dipole were built on both sides of a Rogers RO4003 substrate with a copper thickness of $17 \mu \mathrm{m}$. Then, a piece of F4BM-2 substrate without copper was located in the interlayer. In order to simplify the fabrication, the technology of metallized through hole was employed. Firstly, metal vias with a diameter of $3.3 \mathrm{~mm}$ were fabricated at the same location of each substrate and a little copper at both ends of the metal hole was retained. Then, the three substrates were tightly connected with four plastic screws at the corner of three substrates. The inner core and the outer core of the coaxial line were respectively formed by an SMA feeder and a metal through hole with a diameter of $2.4 \mathrm{~mm}$. The simulated and measured return loss $\left(\mathrm{S}_{11}\right)$ and AR of the designed antenna are shown in Fig. 6. The impedance bandwidth $\left(\mathrm{S}_{11}<-10 \mathrm{~dB}\right)$ reaches $500 \mathrm{MHz}(2.29-2.79 \mathrm{GHz})$ and the AR bandwidth $(\mathrm{AR}<3 \mathrm{~dB})$ achieves $100 \mathrm{MHz}(2.42-2.52 \mathrm{GHz})$. It can be seen that the operating frequency of the 
antenna covers the Rx band $(2492 \pm 8 \mathrm{MHz})$ of the BDS. The radiation patterns are presented in Fig. 7, which was right-hand circularly polarized (RHCP) with very broad CP beamwidth radiation in both the $x z$ - and $y z$-planes. AR versus theta angle of the antenna at $2.492 \mathrm{GHz}$ is shown in Fig. 8(a). At $2.492 \mathrm{GHz}$, the measured results are in good agreement with the simulated ones. The 3-dB AR beamwidth is $135^{\circ}$ for $x z$-plane and $140^{\circ}$ for $y z$-plane. The measured and simulated broadside gain is illustrated in Fig. 8(b). The measured broadside gain was $7.5 \pm 0.2 \mathrm{dBic}$ within the 3-dB AR bandwidth, which was slightly lower than the simulation one. Table I shows the measured performance comparison of the proposed antenna and the previous broad CP antennas. It can be seen that the proposed antenna owns an amazing profile $\left(0.054 \lambda_{0}\right)$.

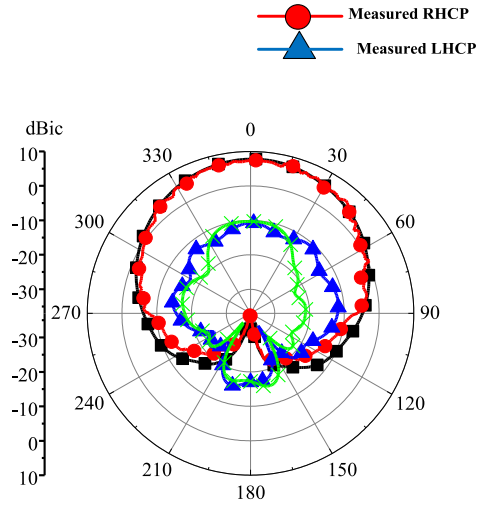

(a)

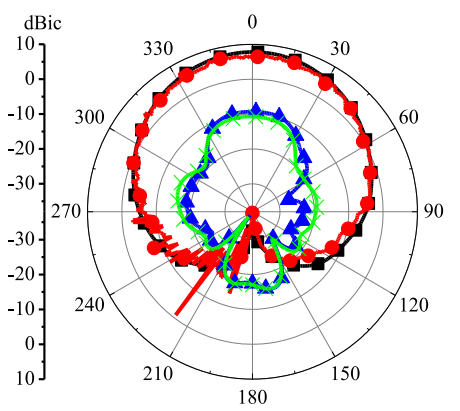

(b)

Fig. 7. Measured and simulated radiation pattern at $2.492 \mathrm{GHz}$ : (a) in $x z$-plane (b) in $y z$-plane.

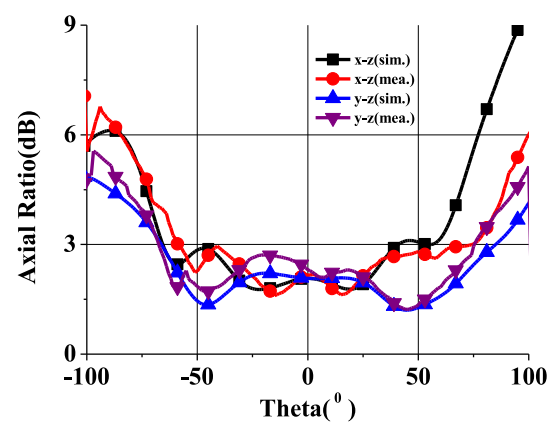

(a)

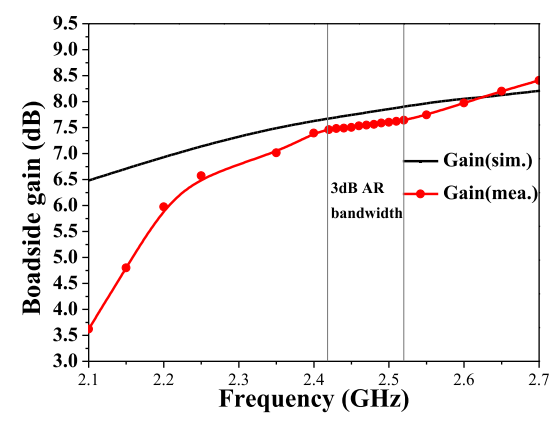

(b)

Fig. 8. Measured and simulated (a) AR versus theta angle at $2.492 \mathrm{GHz}$ (b) broadside gain.

Table I. Measured performance comparison of the proposed antenna and the previous broad CP antennas

\begin{tabular}{|c|c|c|c|c|}
\hline \multirow{2}{*}{$\begin{array}{l}\text { Antenna } \\
\text { structure }\end{array}$} & \multicolumn{2}{|c|}{ 3-dBAR beamwidth } & \multirow{2}{*}{ Feed types } & Size $\left(\lambda_{0}{ }^{3}\right)$ \\
\cline { 2 - 3 } & $\mathrm{x}-\mathrm{Z}$ & $\mathrm{y}-\mathrm{z}$ & & \\
\hline Proposed & $>135^{\circ}$ & $>140^{\circ}$ & single & $0.74 \times 0.74 \times 0.054$ \\
\hline Ref. [4] & $>165^{\circ}$ & $>160^{\circ}$ & single & $0.72 \times 0.72 \times 0.18$ \\
\hline Ref. [5] & $\sim 120^{\circ}$ & $\sim 100^{\circ}$ & single & $0.88 \times 0.88 \times 0.19$ \\
\hline Ref. [9] & $\sim 120^{\circ}$ & $\sim 120^{\circ}$ & dual & $\pi \times 0.37^{2} \times 0.44$ \\
\hline
\end{tabular}




\section{Conclusion}

An AMC-based crossed dipole antenna with low profile and wide-beam RHCP radiation characteristics was presented. It has advantages of broad $\mathrm{CP}$ beamwidth radiation $\left(>135^{\circ}\right)$ and a low profile feature $\left(0.054 \lambda_{0}\right)$. Moreover, the proposed antenna had high front-to-back ratio and stable radiation patterns across the operating bandwidth. Because of these outstanding features, the proposed antenna has a great potential for BDS applications.

\section{Acknowledgments}

Your acknowledgements to co-workers and financial sponsors are placed here. This work was partly supported by the National Science Foundation of China under grant 61401506, the Open Project Program of the State Key Laboratory of Millimeter Waves under Grant No. K201715, Southeast University, and partly supported by the China Postdoctoral Science Foundation, 2016M600349. 